\title{
Effets de différents niveaux d'apport en protéines sur les performances de croissance du lapin de race locale
}

\author{
GP Gongnet 1, M Assane 2, D Dezoumbe 3 \\ 1 Département de zootechnie-alimentation; \\ 2 Département de physiologie-pharmacodynamique, école inter-Etats des sciences \\ et médecine vétérinaires, BP 5077 Dakar, Sénégal; \\ 3 Service de zootechnie-nutrition, Laboratoire de recherches vétérinaires \\ et zootechniques de Farcha, BP 433, N'Djamena, Tchad
}

(Reçu le 27 mars 1992; accepté le 1er novembre 1992)

Résumé - L'influence de différents niveaux d'apport en protéines sur la croissance a été étudiée sur 30 lapins ( 15 mâles et 15 femelles). Les animaux ont été répartis en 3 lots de 10 lapins chacun (5 mâles et 5 femelles) dont les poids moyens respectifs sont de $802,84 \mathrm{~g}$ (lot A), $942,64 \mathrm{~g}$ (lot B) et $917 \mathrm{~g}$ (lot C). Les animaux ont reçu pendant $28 \mathrm{j}$ des aliments dont la teneur en protéines est respectivement de $16,82 \%, 18,20 \%$ et $23,32 \%$ pour les lots A, B et C. La consommation d'aliments diffère significativement entre les 3 lots $(P=0,0076)$ de même que la consommation d'eau par lapin et par jour $(P=0,0144)$. En revanche, l'effet «semainem n'est significatif ni sur les quantités d'eau ingérées par quantités de matière sèche, ni sur l'indice de consommation de 3 lots. Selon les moyennes par cage observées, chaque semaine la vitesse de croissance des lapins varie de manière significative entre les 3 lots $(P=0,0090)$, en ce sens que le lot $B$ se distingue significativement des 2 autres lots $(B>C=A)$.

protéines / consommation d'eau et d'aliment / indice de consommation / lapin / race locale / Tchad

Summary - Effect of different levels of protein on growth performance of local breed rabbits. Three groups ( $A, B$ and $C$ ) of 10 rabbits per group with well established weight were used in this experiment. During the 28-d experimental period, the different groups were fed with rations containing respectively 16.82 (A), 18.20 (B) and $23.32 \%(C)$ protein. The feed intake differed significantly between the 3 groups $(\mathrm{P}=0.0076)$, as did water intake per rabbit per day $(\mathrm{P}=0.0144)$. The "week" effect was not significant either on the amount of water intake or the amount of dry matter and feed conversion in the 3 groups. The average growth rate of rabbits from each group during the experiment varied significantly $(\mathrm{P}=0.0090)$. 


\section{INTRODUCTION}

Les études qui ont été menées sur les besoins du lapin en protéines ont très souvent abouti à des résultats contradictoires. Selon Lebas (1973), Colin (1974), les meilleurs résultats sont obtenus avec des rations alimentaires contenant 17 à $18 \%$ de protéines. Par contre, pour Heckmann et Mehner (1970), Poismans et Wittouck (1986) l'optimum de croissance est atteint avec des rations contenant 27 à $28 \%$ de protéines. Ces résultats quelque peu contreversés nous ont amenés à étudier l'effet de différents niveaux d'apport protéique sur les performances de croissance du lapin de race locale en milieu tropical chaud.

\section{MATÉRIEL ET MÉTHODES}

\section{Animaux}

Trente lapins de race locale, nés entre la fin octobre et la mi-novembre 1988 et entretenus au laboratoire de recherches vétérinaires et zootechniques de Farcha (Tchad) (15 mâles et 15 femelles) ont été utilisés dans l'essai.

Les animaux étaient âgés de 5 mois en moyenne en début d'expérimentation, qui s'est déroulée du 28 mars au 24 avril 1989 au dit laboratoire, et pesaient en moyenne $900 \mathrm{~g}$. Les lapins sont répartis en 3 lots de 10 animaux chacun comprenant 5 mâles et 5 femelles. Chaque lot était maintenu dans des cages de $100 \mathrm{~cm}$ de côté, dont le fond est en grillage d'environ $1 \mathrm{~cm}$ de maille.

Au cours de la période antérieure à l'expérimentation, les lapins ont été nourris à la paille composée essentiellement de Panicum maximum plus une poignée de sorgho blanc concassé.

\section{Aliments et alimentation}

Avant le démarrage de l'essai, les lapins ont été nourris pendant 2 semaines d'une ration de base, dont la composition est consignée dans le tableau I. Les animaux ont été ensuite répartis en 3 lots. Ils étaient alimentés pendant 28 j avec 3 types de rations dont la teneur en protéine varie selon l'apport en farine de Tilapia galillea (tableau II). La composition chimique des 3 types de rations est présentée dans le tableau III. Les aliments et l'eau étaient distribués à voIonté. Chaque jour les quantités aussi bien d'aliments que d'eau refusées étaient déterminées. La différence entre les quantités distribuées et les quantités refusées nous donnait les quantités consommées quotidiennement. Chaque lapin était pesé une fois par semaine.

\section{Analyses statistiques}

Les analyses statistiques des performances de croissance sont réalisées par l'analyse de variance test $F$.

Tableau I. Composition de la ration de base (pourcentage).

Sorgho concassé

75,8

Farine de sang

Feuilles d'Acacia raddina

7,6

Fanes d'arachides

2,5

Sel $(\mathrm{NaCl})$

11,5

Oligo-éléments - Vitamines *

2,3

Coccibio-vit

0,2

0,1

Total

100,0

\footnotetext{
- Olivitasol (formule unitaire); vitamine A (250 000 UI); vitamine D3 (64 $000 \mathrm{UI})$; vitamine E (50 mg); vitamine B1 chlorhydrate (30 mg); vitamine B2 phosphate de sodium ( $80 \mathrm{mg})$; vitamine $\mathbf{B 6}$ chlorhydrate $(30 \mathrm{mg})$; vitamine PP (50 mg); vitamine C (500 mg); pentothénate de calcium (20 mg); lysine mono-chlorhydrate (525 $\mathrm{mg}$ ); sulfate de zinc (10 mg); sulfate de cuivre (2 mg); sulfate de fer (20 mg); sulfate de manganèse (285 $\mathrm{mg}$ ); sulfate de cobalt $(1,5 \mathrm{mg})$.
} 
Tableau II. Composition des aliments des différents groupes (pourcentage).

Groupes

\begin{tabular}{lrrr} 
& A & \multicolumn{1}{c}{ B } & \multicolumn{1}{c}{$C$} \\
\hline & & & \\
Ration de base * & 94,90 & 87,73 & 82,93 \\
Huile de coton & 5,10 & 2,45 & 0,00 \\
Poisson (Tilapia galillea) & 0,00 & 9,82 & 17,07 \\
Total & 100 & 100 & 100 \\
\hline
\end{tabular}

* Voir tableau 1.

Tableau III. Composition chimique des aliments utilisés.

Groupes

A B $C$

$\begin{array}{lrrr}\text { Matière sèche (\% MF) } & 93,9 & 93,9 & 93,8 \\ \text { Matières org (\% MS) } & 86,9 & 82,5 & 78,8 \\ \text { Protéines brutes (\% MS) } & 16,8 & 18,2 & 23,3 \\ \text { Cellulose brute (\% MS) } & 6,6 & 7,7 & 7,1 \\ \text { Extractif non azoté (\% MS) } & 62,7 & 55,5 & 43,1 \\ \text { Matière grasse (\% MS) } & 0,8 & 1,1 & 5,3 \\ \text { Matière minérale (\% MS) } & 7,0 & 11,4 & 15,0 \\ \text { Calcium (\% MS) } & 0,3 & 0,5 & 0,6 \\ \text { Phosphore (\% MS) } & 0,1 & 0,4 & 0,7\end{array}$

MF : matière fraiche séchée à l'air; MS : matière sèche.

\section{RÉSULTATS ET DISCUSSION}

Dans cet essai, les meilleures performances de croissance ont été obtenues sur les lapins recevant une ration contenant $18 \%$ de protéines (tableau IV). Ces résultats sont en conformité avec ceux de Lebas (1973) et Colin (1974), mais diffé- rents de ceux de Poismans et Wittock (1986), Lebas et Ouhayoun (1987) et de Abdella et al (1988) pour lesquels les meilleures performances ont été réalisées avec des rations contenant respectivement 27,21 et $20 \%$ des protéines. Heckmann et Mehner (1970) et Lebas et Ouhayoun (1987) constatent également une amélioration de l'indice de consommation lorsque le taux de protéines passe respectivement de 18 à $28 \%$ et de 6 à $21 \%$.

La différence entre nos résultats et ceux des autres auteurs serait probablement liée à la composition des aliments. En effet, Poisman et Wittouck (1986) ont constaté qu'une forte élévation du taux de protéines alimentaires (27\%) associée à un niveau élevé de cellulose $(12 \%)$ entraîne une amélioration de la croissance et de l'indice de consommation par rapport à une ration contenant $18 \%$ de protéines. Or, dans notre cas, le niveau de cellulose se situe à $7 \%$.

Cependant, d'autres facteurs pourraient expliquer également la disparité de nos résultats avec ceux des autres auteurs. Parmi ces facteurs, on peut citer l'âge (la plupart de ces auteurs ont eu à travailler sur des lapins beaucoup plus jeunes que les nôtres), les sources de protéines et la race. Prenant en compte l'effet des niveaux de protéines et des semaines, il nous a été donné de noter que la consommation d'aliment diffère significativement entre les 3 lots $(P=0,0076)$, de même que la consommation d'eau par animal et par jour $(P=0,0144)$. Cela nous permet de constater que pour ces 2 paramètres, le lot $A$ se distingue des 2 autres $(\mathrm{A}<\mathrm{B}=\mathrm{C})$. En revanche, même en tenant compte de l'effet "semaine", ni la proportion d'eau par matière sèche, ni l'indice de consommation ne varient significativement entre les 3 lots. Le même calcul effectué à partir des moyennes par cage observées chaque semaine montre 
Tableau IV. Effets de différents nivaux d'apport en protéines sur la consommation d'aliments et d'eau, le gain moyen quotidien (GMQ) et l'indice de consommation (IC) du lapin.

\begin{tabular}{|c|c|c|c|c|c|}
\hline Semaines & $\begin{array}{c}\text { Aliment } \\
(g M S / L * / J)\end{array}$ & $\underset{\left(m / / L^{*} / j\right)}{E a u}$ & $\begin{array}{c}\text { Eau/aliment } \\
\text { (ml/gMS) }\end{array}$ & $\begin{array}{c}\text { GMQ } \\
\left(g d^{\prime} \text { alim } /\right)\end{array}$ & IC \\
\hline \multicolumn{6}{|l|}{ Groupe A } \\
\hline $1^{\text {re }}$ semaine & 33,1 & 164,8 & 4,9 & 9,8 & 3,4 \\
\hline $2^{\ominus}$ semaine & 48,5 & 228,3 & 4,7 & 14,8 & 3,2 \\
\hline $3^{\theta}$ semaine & 41,7 & 207,6 & 4,9 & 11,1 & 3,7 \\
\hline $4^{e}$ semaine & 41,2 & 200,1 & 4,8 & 9,6 & 4,2 \\
\hline Moyenne & 41,1 & 200,2 & 4,8 & 11,3 & 3,6 \\
\hline SE & 5,4 & 22,9 & 0,1 & 2,1 & 0,4 \\
\hline \multicolumn{6}{|l|}{ Groupe B } \\
\hline $1^{\text {re }}$ semaine & 40,3 & 164,3 & 4,1 & 16,9 & 3,6 \\
\hline $2^{\circ}$ semaine & 60,7 & 300,0 & 4,9 & 16,4 & 3,7 \\
\hline $3^{e}$ semaine & 53,6 & 310,7 & 5,8 & 15,4 & 3,5 \\
\hline $4^{e}$ semaine & 60,5 & 314,6 & 5,2 & 16,1 & 3,7 \\
\hline Moyenne & 53,7 & 272,4 & 5,0 & 16,2 & 3,3 \\
\hline SE & 8,3 & 62,6 & 0,6 & 0,5 & 0,5 \\
\hline \multicolumn{6}{|l|}{ Groupe C } \\
\hline $1^{r e}$ semaine & 36,5 & 183,1 & 5,0 & 12,9 & 2,8 \\
\hline $2^{e}$ semaine & 61,2 & 297,3 & 4,8 & 14,5 & 4,2 \\
\hline $3^{\theta}$ semaine & 60,4 & 303,6 & 5,0 & 14,1 & 4,2 \\
\hline $4^{e}$ semaine & 55,4 & 289,7 & 5,3 & 10,5 & 5,1 \\
\hline Moyenne & 53,4 & 268,4 & 5,0 & 13,0 & 4,1 \\
\hline$S E$ & 9,9 & 49,5 & 0,2 & 1,5 & 0,8 \\
\hline
\end{tabular}

L : lapin.

que la vitesse de croissance varie bien de manière très significative entre les 3 lots $(P=0,0090)$ et que cette fois, le lot $\mathrm{B}$ se distingue significativement des 2 autres lots $(B>C=A)$.

Les lapins du groupe $C$ sont affaiblis et amaigris vers la fin de la $4^{e}$ semaine. Dans la même période, des dépilations ont été observées sur la plupart des animaux de ce groupe. Aucun cas de mortalité n'a été noté au cours de la période d'expérience.

\section{RÉFÉRENCES}

Abdella HM, Shalash SMM, Boulos NZ, Selm $A D$ (1988) Effect of growing rabbits of feeding different levels of crude protein. $J$ Appl Rabbit Res 11 (4), 252-256

Colin M (1974) Supplémentation en lysine d'un régime de base de tourteau de sésame chez le lapin. Effets sur les performances de croissance et le bilan azoté estimé par 2 méthodes. Ann Zootech 23, 119-132

Heckmann FW, Mehner A (1970) Versuch über den Eiwess und Rohfasergehalt im Alleinfut- 
ter für Fungmastkaninchen. Arch Geflügelzucht Kleintierk 19, 29-43

Lebas $F(1973)$ Effets de la teneur en protéines de rations à base de soja ou de sésame sur la croissance du lapin. Ann Zootech 22, 8392

Lebas F, J Ouhayoun (1987) Incidence du niveau protéique de l'aliment, du milieu d'élevage et de la saison sur la croissance et les qualités bouchères du lapin. Ann Zootech 36 (4), 421-432

Poismans R, Wittouck PJ (1986) Effets d'une ration riche en protéines et pauvre en cellulose sur les performances de croissance, de développement des organes et la composition de la carcasse chez le lapin blanc de Termonde. Ann Zootech 35 (1), 6178 